\title{
Ultra-high-energy cosmic rays and neutrinos from tidal disruptions by massive black holes (Corrigendum)
}

\author{
Claire Guépin $^{1,2,3}$, Kumiko Kotera ${ }^{1}$, Enrico Barausse ${ }^{1,4,5,6}$, Ke Fang $^{7}$, and Kohta Murase $8,9,10,11$ \\ ${ }^{1}$ Sorbonne Université, CNRS, UMR 7095, Institut d'Astrophysique de Paris, 98 bis bd Arago, 75014 Paris, France \\ e-mail: claire.guepin@iap.fr \\ 2 Department of Astronomy, University of Maryland, College Park, MD 20742, USA \\ Joint Space-Science Institute, University of Maryland, College Park, MD 20742, USA \\ 4 SISSA, Via Bonomea 265, 34136 Trieste, Italy \\ 5 INFN Sezione di Trieste, Trieste, Italy \\ ${ }^{6}$ Institute for Fundamental Physics of the Universe, Via Beirut 2, 34014 Trieste, Italy \\ Kavli Institute for Particle Astrophysics and Cosmology, Stanford University, Stanford, CA 94305, USA \\ 8 Department of Physics, The Pennsylvania State University, University Park, PA 16802, USA \\ 9 Department of Astronomy \& Astrophysics, The Pennsylvania State University, University Park, PA 16802, USA \\ ${ }_{10}$ Center for Particle and Gravitational Astrophysics, The Pennsylvania State University, University Park, PA 16802, USA \\ 11 Yukawa Institute for Theoretical Physics, Kyoto 606-8502, Japan
}

A\&A, 616, A179 (2018), https://doi .org/10.1051/0004-6361/201732392

Key words. astroparticle physics - neutrinos - errata, addenda

We noticed a normalization error in our calculations and a bug in our intergalactic propagation code, which impacted our predictions of the ultra-high-energy cosmic ray (UHECR) and highenergy (HE) neutrino fluxes from jetted tidal disruption events (TDE; Guépin et al. 2018). Correcting these errors led to sizable changes in the cosmic rays and neutrino fluxes, but our conclusions remain the same.

The corrected UHECR and HE neutrino spectra from single TDEs, in the high and medium states, are illustrated in Figs. 1 and 2. The high state of the UHECR spectrum is slightly modified as the maximum energy of injected particles is determined by the competition between the acceleration and the dynamical timescales, and not between the acceleration and the cooling timescales. As stated above, the HE neutrino fluxes are one order of magnitude higher that the ones derived previously. Thus we show that the HE neutrino flux from one source at $d_{L, 2}=50 \mathrm{Mpc}$ reaches the IceCube detection limit. We note that considering the observed event rate density of jetted TDE is $\sim 0.03 \mathrm{Gpc}^{-3} \mathrm{yr}^{-1}$, the rate of TDEs at distances smaller than $50 \mathrm{Mpc}$ is $4 \times 10^{-6} \mathrm{yr}^{-1}$, which is extremely low. The sensitivity limit of GRAND has also been updated (GRAND Collaboration 2020) as GRAND cannot observe a TDE during its entire duration: The source moves out of the field of view of the detector because of the Earth's rotation. Thus the GRAND sensitivity limit for long duration bursts (lasting for more than a few hours) is reduced when compared to the sensitivity limit for short duration bursts, which was previously shown.

The corrected diffuse UHECR and HE neutrino spectra, as well as the corresponding UHECR composition, are illustrated in Figs. 3 and 4. The bug in the intergalactic propagation code only affected the spectrum of high-energy protons. A large number of protons pile up below $10^{19} \mathrm{eV}$. It affects the composition of UHECR, which better reproduces, than our precious results, the standard deviation of the atmospheric depth of air shower maximum development $\sigma\left(X_{\max }\right)$ observed by the Pierre Auger observatory below $10^{19} \mathrm{eV}$.

The fraction $f_{\mathrm{s}}$ of the total population of TDE required to reproduce the data from the Pierre Auger observatory is modified. The diffuse UHECR spectrum is calculated for an injection of $70 \% \mathrm{Si}$ and $30 \% \mathrm{Fe}$, a spectral index $\alpha=1.5$, an acceleration efficiency $\eta_{\text {acc }}=0.1$, a TDE source evolution, and a fraction $\xi_{\mathrm{CR}} f_{\mathrm{s}}=2.6 \times 10^{-3}$ of the local event rate density $\dot{n}_{\text {tde }, 0}=1.5 \times 10^{2} \mathrm{Gpc}^{-3} \mathrm{yr}^{-1}$. This rate is computed from the TDE rate per galaxy obtained in the case of core galaxies. The population fraction approximately corresponds to the rate density $\sim 0.4 \mathrm{Gpc}^{-3} \mathrm{yr}^{-1}$. Moreover, the predicted diffuse HE neutrino spectrum is lower than in our previous estimates, as our estimate of $f_{\mathrm{s}}$ has decreased, and is about two orders of magnitude below the spectrum of HE neutrinos detected by IceCube. The detection of very-high-energy neutrinos from TDE by detectors, such as GRAND, also seems disfavored.

From these results, accounting for time variability of multimessenger emissions seems to be important as the bulk of the different messengers are not typically emitted during the same phase. In this example, most of the UHECR contributing to the diffuse flux, allowing the Auger data to be reproduced, are produced during the medium state, whereas most of the HE neutrinos are produced during the high state. In order to reproduce the Auger data, a model accounting only for the high state would require a higher baryon loading or a higher TDE event rate, which would subsequently increase the associated HE neutrino flux. 

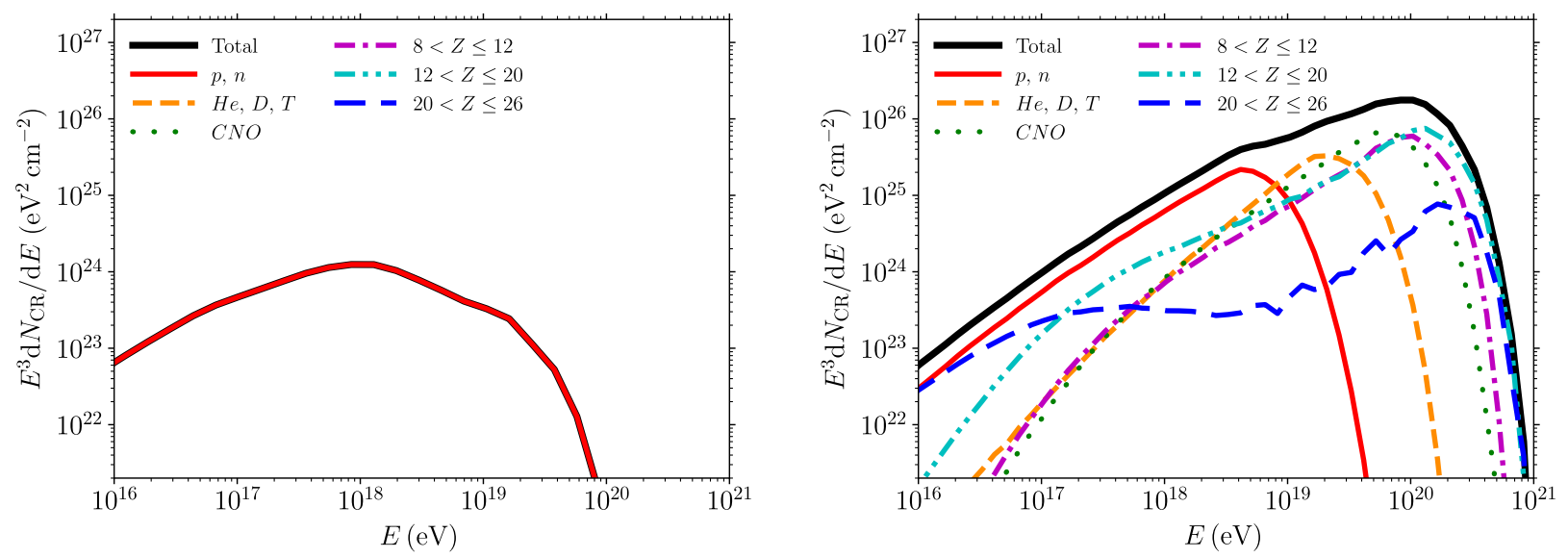

Fig. 1. Cosmic-ray spectra for one source with pure iron injection with spectral index $\alpha=1.8$, photon field with $\hat{a}=0.07$, and acceleration efficiency $\eta_{\text {acc }}=0.2$. We show the total spectrum (black) and the composition (other colors), as indicated in the legend, for TDE around a black hole of mass $M_{\mathrm{bh}}=7 \times 10^{6} M_{\odot}$, with a corresponding SED in its high state with $L_{\mathrm{pk}}=10^{47.5} \mathrm{erg} \mathrm{s}^{-1}$ and $t_{\mathrm{dur}}=10^{5} \mathrm{~s}$ (left) and in its medium state with $L_{\mathrm{pk}}=10^{46} \mathrm{erg} \mathrm{s}^{-1}$ and $t_{\mathrm{dur}}=10^{6} \mathrm{~s}(r i g h t)$ for a source distance $d_{L, 1}=1.88 \mathrm{Gpc}$. Here, we assume $\xi_{\mathrm{CR}}=1$.
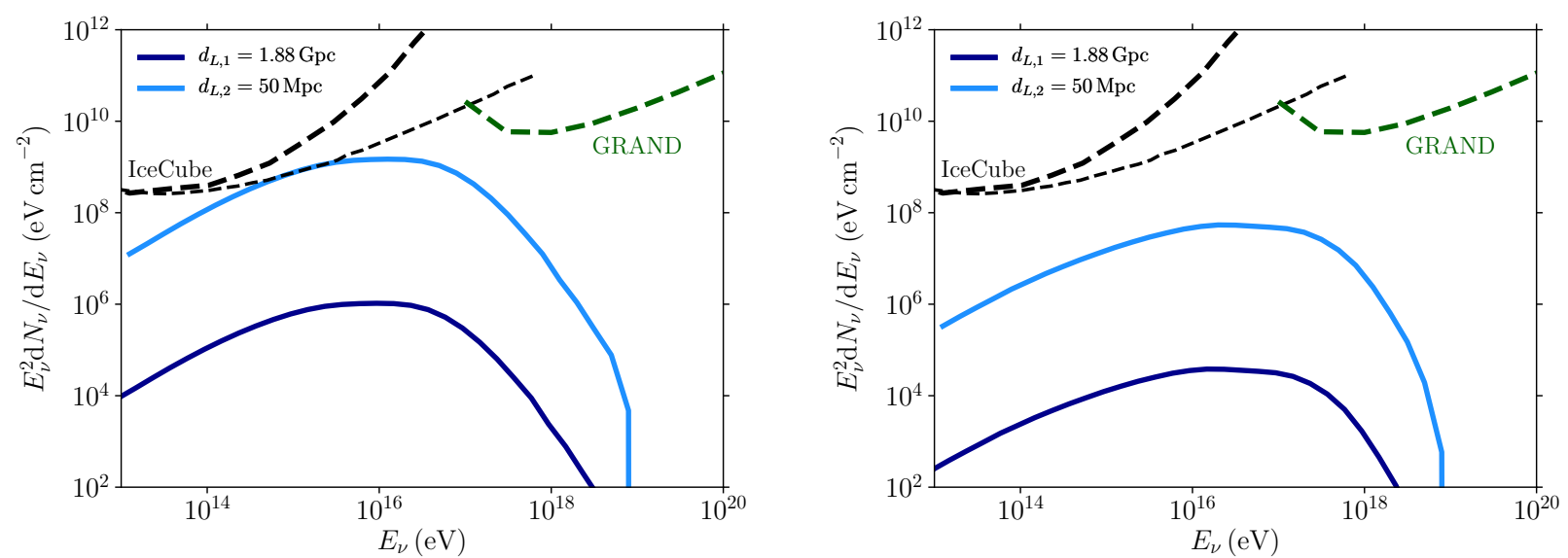

Fig. 2. Neutrino spectra for three flavors for one source with same characteristics as in Fig. 1. We show the total spectra (in $\mathrm{eV} \mathrm{cm}^{-2}$ ) for a high state SED with $L_{\mathrm{pk}}=10^{47.5} \mathrm{erg} \mathrm{s}^{-1}$ (left) and a medium state SED with $L_{\mathrm{pk}}=10^{46} \mathrm{erg} \mathrm{s}^{-1}$ (right). We consider two different distances $d_{L, 1}=1.88 \mathrm{Gpc}$ (dark blue) and $d_{L, 2}=50 \mathrm{Mpc}$ (light blue). The IceCube and projected GRAND (GRAND Collaboration 2020) sensitivities are also shown (dashed black and green lines). For the IceCube sensitivities, we show two cases depending on the declination: $0^{\circ}<\delta<30^{\circ}$ (most favorable case, thin line) and $30^{\circ}<\delta<60^{\circ}$ (Swift J1644+57 case, thick line) (Aartsen et al. 2014).
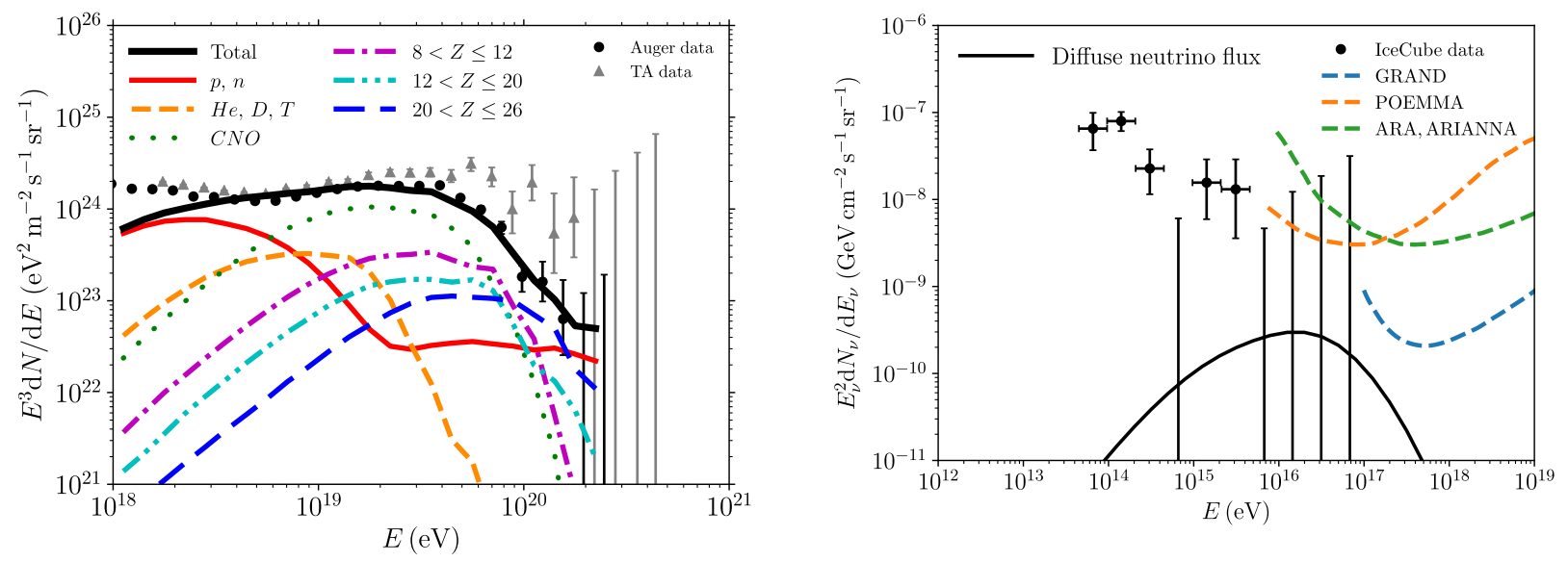

Fig. 3. Diffuse cosmic-ray (left) and neutrino (right) spectra from a population of jetted TDEs obtained for an injection of $70 \%$ Si and $30 \%$ $\mathrm{Fe}$, with spectral index $\alpha=1.5, \xi_{\mathrm{CR}} f_{\mathrm{s}}=2.6 \times 10^{-3}$ and source evolution derived in this work, with maximum bolometric luminosity in the population $L_{\max }=6.8 \times 10^{46} \mathrm{erg} \mathrm{s}^{-1}$ (in the medium state). Left: total spectrum (black) and its composition. We superimposed data from the Auger experiment (black dots, The Pierre Auger Collaboration 2015) and from the Telescope Array experiment (gray dots, Fukushima 2015) for which only statistical uncertainties are shown. Right: diffuse neutrino flux for three flavors (black), the diffuse neutrino flux measured by the IceCube experiment (IceCube Collaboration 2017), and the projected limits for GRAND (GRAND Collaboration 2020), ARA/ARIANNA (Allison et al. 2015; Barwick et al. 2015), and POEMMA (Neronov et al. 2017). 
C. Guépin et al.: Ultra-high-energy cosmic rays and neutrinos from tidal disruptions (Corrigendum)
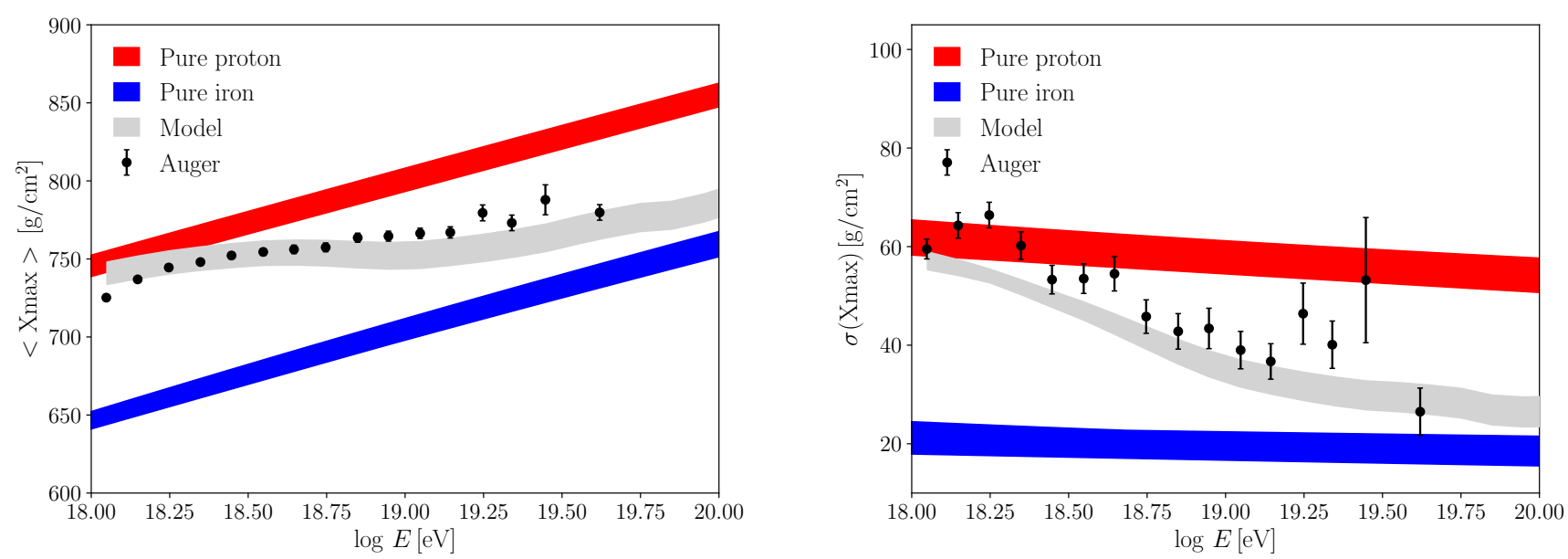

Fig. 4. Mean and standard deviation of $X_{\max }$ for the spectrum shown in Fig. 3 (pale gray band). We also show Auger measurements (Aab et al. 2014) with uncertainties (black dots) and simulation results for pure proton injection (red band) and pure iron injection (blue band). The bands are obtained by accounting for hadronic model uncertainties (EPOS-LHC, SIBYLL 2.1 or QGSJET II-04).

\section{References}

Aab, A., Abreu, P., Aglietta, M., et al. 2014, Phys. Rev. D, 90, 122005 Aartsen, M. G., Ackermann, M., Adams, J., et al. 2014, ApJ, 796, 109

Allison, P., Auffenberg, J., Bard, R., et al. 2015, Astropart. Phys., 70, 62

Barwick, S. W., Berg, E. C., Besson, D. Z., et al. 2015, Astropart. Phys., 70, 12

Fukushima, M. 2015, Eur. Phys. J. Web Conf., 99, 04004
GRAND Collaboration (Alvarez-Muniz, J., et al.) 2020, Sci. China Phys. Mech. Astron., 63, 219501

Guépin, C., Kotera, K., Barausse, E., Fang, K., \& Murase, K. 2018, A\&A, 616, A179

IceCube Collaboration (Aartsen, M.G., et al.) 2017, ArXiv e-prints [arXiv:1710.01191]

Neronov, A., Semikoz, D. V., Anchordoqui, L. A., Adams, J. H., \& Olinto, A. V. 2017, Phys. Rev. D, 95, 023004

The Pierre Auger Collaboration (Aab, A., et al.) 2015, ArXiv e-prints [arXiv:1509.03732] 